Voix et Images

\title{
Petite conversation de taverne. Entretien (ou ce qui en tient lieu)
}

\section{Louis Hamelin}

Volume 13, numéro 3 (39), printemps 1988

Jack Kerouac et l’imaginaire québécois

URI : https://id.erudit.org/iderudit/200725ar

DOI : https://doi.org/10.7202/200725ar

Aller au sommaire du numéro

Éditeur(s)

Université du Québec à Montréal

ISSN

0318-9201 (imprimé)

1705-933X (numérique)

Découvrir la revue

\section{Citer ce document}

Hamelin, L. (1988). Petite conversation de taverne. Entretien (ou ce qui en tient lieu). Voix et Images, 13(3), 380-387. https://doi.org/10.7202/200725ar d'utilisation que vous pouvez consulter en ligne.

https://apropos.erudit.org/fr/usagers/politique-dutilisation/ 


\section{Petite conversation de taverne. Entretien (ou ce qui en tient lieu)}

\section{par Louis Hamelin, Université du Québec à Montréal}

NDLR: conversation renregistrée» lors du passage de Carolyn Cassady à Montréal en octobre 1987, quelques jours après la Rencontre internationale Jack Kerouac, tenue à Québec. En réalité, il s'agit d'un monologue, comme le sont souvent les conversations d'inspiration éthylique. Celui-ci a beaucoup plu à Carolyn Cassady: Il n'a pas seulement compris, disait-elle, il pense comme Jack.

La légendaire Carolyn Cassady (épouse de feu Neal Cassady) assise à une table bancale; le vieux Maurice revient, les mains pleines de Black Label romantiques (rouges et noires). La musique est tonitruante (pas moyen de disserter en paix), les éclairages tour à tour fuligineux et fulgurants s'entrecroisent dans l'obscurité habitée par des chorégraphies de nymphes si expressives...

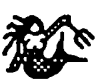

«Voilà, Carolyn, je veux te parler, je ne veux point te cruiser, te parler de la solitude, de la paix intérieure que je ne connais pas et qui n'est imaginable qu'à des années-lumière de la débauche polychrome de cet endroit. Je veux te parler de la paix solitaire de Jack Kerouac, celle qu'il a approchée, qu'il n'a pas su conserver parce qu'il préférait, au fond, la route à finir aux cabanes de sa vie. Je veux te parler de Jack pour mieux te parler de moi (solipsisme oblige) et montrer que lorsque nous considérons Jack Kerouac redevenu enfant dans les bois, Jack-in-the cabin qui est un ange, les diablotins ne sont jamais loin. Hypothèse, Carolyn (puisqu'il en faut une, même ici): Desolation Angels 1 et Big Sur ${ }^{2}$ constituent les pôles éloignés du périple kérouacien. Desolation Angels, à l'apogée du mouvement ascendant, contient l'aspiration pure de l'âme à l'éternité. Big Sur, tout au bout de la descente aux enfers, marque la condamnation irrévocable du corps humain à la mort. Alors, reportons-nous loin là-bas, dans l'Ouest mythique de nos rêves, sur les pics enneigés qui se pressent dans une fantastique chevauchée orogénétique et onirique, tout contre la frontière du Canada. Tu es là, Jack? Oh yeah you bet, he says. Ou bien, Ben oui, c't'affaire. Il a gravi la pente

1 Jack Kerouac, Desolation Angels, Frogmore, St. Albans, Herts, Panther Books Ltd, 1972 (1966 C)), 366 p.

2 Jack Kerouac, Big Sur, Paris, Gallimard, coll. «Folio», 1966 (1962 (c)), 308 p. 
abrupte en compagnie des ânes rétifs et de vieux forestiers experts de ces contrées montueuses. Puis te voilà seul, Jack Kerouac, seul sans une once de pinard pour dessécher ton viril gosier réduit au monologue. Écoute, Carolyn, passe-moi une autre Black Label et laisse-moi te dire ceci (mais tu le sais déjà): Kerouac à Desolation Peak, c'est l'expérience absolue du Vide. Kerouac savait par ses lectures que le Vide est en toute chose (et moi, je n'y connais rien à ces chinoiseries), mais là-haut, Jack se trouve devant le Vide projeté dans le paysage. Le mont Hozomeen, c'est le Vide. Il y a osmose entre l'homme et le rude pays: $I$ will be the Void, moving without having moved ${ }^{3}$. Remarque une chose: ce vieux Jack s'ennuie, bien sûr, il préférerait de loin se retrouver en bas, avec les bums ailés de la côte, dans les bouges sanctifiés d'où s'élève la poésie renaissante de la nuit américaine. Mais la révélation du Vide lui impose la paix; Hozomeen s'érige en modèle de la grande Illusion. I come back into the house a new man 4 . Est-ce le début de la sagesse? Ah, Jack s'est approché là-bas de la perfection terrestre, de la sapience intuitive d'un bonze américain, de son rêve avoué de cesser toute fuite et de devenir le second Thoreau. Il s'est arraché (par besoin pécuniaire, ne l'oublions pas) à la limite du possible, aux forces gravitationnelles qui le tiraient par la manche: $E h$, vieux Jack, viens prendre un coup! Jack fait face au Vide avec un enthousiasme tranquillement jubilatoire. À Desolation Peak, il est tourné vers l'avenir, vers l'Éternité Dorée. Oh je sais, Carolyn, il pense toujours à Mémère et à Maggie, il renoue avec ce jeu de baseball puissamment imaginatif de sa jeunesse, il revoit en songe les murs de brique rouge vin que frôle le Docteur Sax. On ne peut faire autrement, avec la mémoire d'éléphant en voie de disparition qu'il possède. Mais tandis qu'il ressuscite le passé et qu'il manie de sa plume les prosopopées, Jack se projette dans le futur: il songe avec délices aux cuites carabinées sur le sol tremblant de Frisco, aux bouffes chinoises qu'il fera, à la solidarité muette et inentamable de tous les poivrots de la terre. À Desolation Peak, Jack Kerouac vit encore d'espoir, si je puis me permettre.

«Le monde de la bouteille ne lui a pas encore révélé son sinistre cul grossissant qui peut faire naître l'horreur même de l'Éden retrouvé. Sur les cimes éthérées, Kerouac peut encore idéaliser la bohème, la bamboche, les bitures du marin en permission à perpétuité. Carolyn! Je le jure sur ce qui m'est le plus sacré et sur ce qui m'unit à Jack mort et enterré (la certitude de grandir seul dans la nuit du monde, jumelée à l'extase épiphanique de quelques rares éblouissements): Jack atteint presque à la sérénité sur le mont de la Desolation. Desolation est un de ces éblouissements, un pic sur le cardiogramme inégal de la vie. Chaque fois qu'il se sent faiblir et redevenir humain faillible sur le bord du précipice, il peut se tourner vers le mont Hozomeen, sur cette massive et orgueilleuse silhouette qui est aussi vide que sa propre petite vie de pauvre Canuck dévié de sa route migratoire. Quand je pense à Kerouac sur Desolation Peak, à l'anachorète ébloui qui fait le poirier matutinal, qui apprend à considérer le monde à l'envers et pédale dans les nuages de la terre, je pense à un écrivain près de son apogée.

3 Desolation Angels, op. cit., p. 32.

4 Ibid., p. 31. 
Kerouac est au sommet, au propre et au figuré. Dès que Sur la route paraîtra, son cheminement empruntera la longue courbe descendante menant à l'hémorragie terminale dans la cuvette de Mémère, en Floride, la cuvette où Kerouac aura vomi son être divin. Je vais à la pissotière, excuse-moi Carolyn... je reviens.»

(3)

«So, you were saying that Desolation was a peak in Jack's life. But Jack was always Jack...»

«Oui, je sais, même à Desolation Peak, les puissances terrifiantes du monde des ténèbres prennent soin de rappeler leur omnipotence. Peu après son arrivée, le mont Hozomeen apparaît à Kerouac, dans la nuit, comme un donjon d sorcières 5 . Le flanc en terrasse de la montagne, c'est aussi la voie d'une possible descente dantesque. Et il y a l'affaire du «meurtre de la souris», dont la compréhension nous est cruciale. Voici: dans Desolation Angels, Kerouac mentionne, en passant, la liquidation d'une souris, et la tentative d'en tuer une autre. Mais il n'épilogue pas outre mesure sur cet épisode scabreux, puisqu'il relate à ce moment sa révélation du Vide. Curieusement, ce n'est qu'au moment de redescendre, au début de la deuxième partie intitulée «Desolation in the World» 6 que Kerouac battra sa coulpe et acceptera d'analyser l'affaire du «souricide». Nous y reviendrons, si tu veux Carolyn, mais qu'il suffise de noter (et c'est très important) que l'idylle avec la divinité, qui prévaut sur le sommet de Desolation, exclut la culpabilisation complaisante. Si des présages de mort existent, Kerouac refuse pour l'instant de les voir. Les signes funèbres qui fondront de Big Sur épargnent encore la montagne de la certitude absolue de sombrer un jour dans l'origine du mal. Eh, Carolyn! Mon estomac est vide et je manque de sommeil. Enfin, les conditions favorables à la création sont réunies...» reviens...»

«Il faut d'abord (merci, à ta santé) faire le vide (cerveau, estomac). Je

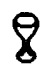

«You were just saying that conditions were right...»

«Ah, oui, je reviens à ce vieux, à ce bon vieux, à cet ange archétypique qui hante les cellules grises de ma nuit ignare: Jack Kerouac. Jack seul sur la montagne; l'ascétisme forcé. Le soin apporté aux plus infimes détails de la vie

5 Jack Kerouac, les Clochards célestes, Paris, Gallimard, coll. «Folio», 1963, p. 357.

6 Le livre I de Desolation Angels comprend les parties «Desolation in Solitude» et «Desolation in the World». Seul le livre II, intitule «Passing Through», a été traduit en français sous le titre les Anges vagabonds. 
quotidienne: balayer le plancher, faire la popote, faire du feu. Parmi les rêves de femmes lascives, les rêves de chute luxuriante, Jack est pris au piège de sa propre aspiration à la paix. En lui, se joue le drame éternel de la destinée humaine, cet écartèlement entre le haut et le bas, Jack-in-the-box!»

$$
\text { «Oh, my...» }
$$

«Jack vit en moi et en toi et en tout le monde par la pérennité de ses contradictions. L'été demier, Carolyn, lorsque je turbinais au pays des Attikamegs, je luttais pour m'imposer une discipline intellectuelle, pour m'astreindre à un confinement vespéral bénéfique dans l'exiguiité de ma chambre où je lisais le reflet de la réalité dans les gros bouquins. Mais j'entendais toujours l'appel de la nuit, l'appel des astres à leur sizygie, l'appel des Indiens et de leurs fantômes, et goûtais l'amertume des bouteilles de bière et la promiscuité des sauvagesses dans les grandes tentes blanches... mais trêve de réminiscences. C'est de Kerouac qu'il faut discourir, même si en disséquant son généreux cadavre littéraire, on découvre des petits morceaux de nous-mêmes. Kerouac est pénétré du Vide rédempteur, de la notion rassurante que Rien n'est rien, ce qui excuse à l'avance les frasques qui salueront le retour à la ville. Il entend l'appel de la bohème d'en bas, l'exhortation à la bamboula. Il affirme avoir acquis sur ses rochers $[. .$.$] la certitude d'une$ liberté de la prairie éternelle?. Mais cette belle vérité chèrement arrachée à la nudité de la prairie alpine ne lui sera d'aucune utilité au niveau de la mer.»

\section{«At Big Sur, of course...»}

«Cette belle vérité, Carolyn, n'est opérante que dans la mesure où elle se nie: Kerouac se libère à Desolation Peak, mais il ne peut y être libre, car il ne s'y trouve nulle occasion de mettre cette liberté à l'épreuve. Et le séjour à Big Sur suffira à prouver que son émancipation sommitale est illusoire, qu'il n'a été sevré que temporairement des attaches terrestres qui se prolongent jusqu'à lui par leur morbide pouvoir de séduction.

«Bon. Il faut résumer le séjour à Desolation Peak. Desolation Peak, c'est l'illumination. Kerouac ne repérera pas de feux de forêt, mais il verra des éclairs: l'illumination est un éblouissement, un éblouissement passager. L'euphorie n'aura qu'un temps. Le mouvement va s'inverser, l'élévation spirituelle se changer en dégringolade infernale. Quand Jack prend congé de l'hospitalité fruste de Desolation Peak, il ne se doute peut-être pas qu'il va descendre longtemps, longtemps, que la débandade vertigineuse va l'emporter, des années plus tard, vers la toute dernière extrémité du continent de ses errances. Le mouvement ascendant ne se prolongera pas dans l'avenir, dans l'éternité. Desolation Peak, c'est le point culminant de la route. À partir de là, pour Old Kerouac, ce sera downhill all the way, jusqu'en Floride, jusqu'à la cuvette de chez Mémère...»

«Downhill all the way...»

$7 \quad$ Les Clochards célestes, op. cit., 372. 
«Comme je t'ai dit, Carolyn, l'épisode du «meurtre» d'une souris prend place dans la cabane de Desolation Peak, mais Kerouac ne s'étend sur le sujet qu'au moment de raconter la redescente, le retour à la disgrâce de la ville où la belle illusion du Vide se dissipera pour faire place à la matérialité brute de l'être de poussière lessivé par la mer. Ả Big Sur, Old Jack se retrouvera enchaîné inexorablement à ses origines (la route tourne en rond, il revient vers la Bretagne de ses ancêtres). La vérité de l'origine, c'est le péché inexpiable. Alors, au moment de retourner vers le monde, le monde non pas libre mais condamné à ses plaisirs morbides, alcool, drogues et prostituées, antichambres de la mort déjà, Kerouac S'ACCUSE. S'accuse d'avoir assassiné une souris et d'avoir toléré l'agonie d'un autre rongeur sans rien tenter. Avant même de confronter sa nouvelle liberté éternelle au monde souterrain des tripots où elle devient caduque, il possède la prescience du péché, de la chute, et il trace sa filiation directe avec Caïn, premier meurtrier de l'histoire de l'humanité, Caïn le berger, le nomade. I had abdicated my position as a holy angel from heaven who never killed...8"

\section{«Yes, Neal tried to teach him Cayce...»}

«La disposition syntagmatique de la confession de ce menu crime, contiguẽ à la redescente vers le pays d'en bas, illustre clairement la portée symbolique de cet incident: l'ange doré est en train de déchoir. La culpabilité kérouacienne, qui prend corps dans la minuscule entité d'un membre de la gent trotte-menue, sera au centre du drame de Big Sur vers lequel nous nous dirigeons implacablement, sur les traces de la plume angélique de Jack Kerouac rappelée par la gravité de la Terre (plume de canard boîteux).

«THIS IS INFERNO. BIG SUR, CAROLYN! «Bornes» in the USA! À Big Sur (attention Carolyn, buvons à l'étiquette noire) le Vide rassurant d'en haut devient une réalité terrifiante. À Desolation Peak, le Vide se trouvait au-dessus de la tête de Jack, appel, aspiration à l'élévation spirituelle. À Big Sur, le Vide s'ouvre sous ses pieds. Il menace d'engloutissement le corps tout entier. L'Illumination de la montagne s'abolit dans les ténèbres: [...] j'allume donc ma lanterne de cheminot pour risquer un ceil timide alentour, mais sa clarté se perd, tout comme celle de la voiture, dans le vide 9 . À Big Sur, la route de Kerouac se termine abruptement, au bord du gouffre. Au fond, il y a la mer qui avale tout: $[. .$. ce merveilleux chemin sableux qui plonge, plonge 10 . Kerouac est atterré par la vision d'une carcasse d'automobile qui gît ensablée après avoir quitté la route pour effectuer le grand saut. C'est une métaphore tragique de la fin. La topographie big-surienne inspire une grande terreur à notre grand Jack. À Desolation Peak, la première nuit, le mont Hozomeen avait de même épouvanté notre ami. Mais par la méditation, par la fusion physique avec les projections glacées de l'univers, Kerouac avait pu apprivoiser le Vide, trois ou quatre années plus tôt. À Big Sur, le Vide se met à rugir, bête fauve redevenue sauvage et destructrice. La

8 Desolation Angels, op. cit., p. 89.

9 Big Sur, op. cit., p. 26.

10 Ibid., p. 23. 
montagne baptisée Mien Mo n'évoque qu'un cauchemar menaçant qui l'écrase. Kerouac descend si bas à cet endroit que la mer se situe au-dessus de lui, avec ses lames aux crêtes effrayantes. Et les signes et présages maléfiques s'accumulent: chauve-souris le frôlant dans la cabane (serait-ce le fantôme de la souris morte?), lecture de Dr. Jekyll and Mr. Hyde qui contient la clé du séjour au chalet de Monsanto, etc. À Big Sur, il n'y a plus d'anges dorés, mais un tête-à-tête avec les démons (un rat court sur sa tête11). Big Sur est un thêâtre de tragédie, un décor pour film d'horreur naturaliste. C'est avant tout l'expérience capitale de la solitude, cet état béni auquel Kerouac a toujours voulu accéder, et qu'il a toujours eu peur d'affronter. Toujours la même histoire, hein Carolyn? Comme dans ce livre de Joyce Johnson ${ }^{12}$, quand Jack fait le væu de passer une semaine seul à Cherry Valley, dans le cottage de Lucien Carr, et dès le mardi soir, le voilà revenu à Big Apple plongé dans la bain de Joyce... ah la la.»

\section{"Vive la différence...»}

"Carolyn, d'accord, tandis que sur le toit du monde, Kerouac se tenait face au Vide et tourné surtout vers l'avenir (tourné, en tout cas, vers un avenir plutôt rose, en même temps que sur son passé d'éléphant hypermnésique), sur la côte du Pacifique, il doit faire face à la mer et fatalement au passé; son passé à lui, mais aussi le passé de la race humaine et le passé géologique de la planète. Il pense avec insistance aux Indiens du $\mathrm{X}^{\mathrm{e}}$ siècle. Même le futur se voit recouvrir par le passé: [...] l'entière surface de ce monde [...] sera peu d peu recouverte du limon d'un milliard d'années ${ }^{13}$. Au lieu de méditer, Old Jack se met à transcrire furieusement les bruits de la mer, à la faveur de la nuit. Par une discrète effraction, il pénètre dans le monde sans lumière des abysses d'où est partie la vie. La mer, insinueusement, semble vouloir contredire les théories bouddhiques. Elle dit: tu crois que tu n'es que Vide? Regarde-moi en face: tu es fait d'eau salée. De fait, à Big Sur, Kerouac me paraît se détourner déjà des préceptes dụ bonhomme Bouddha. Il abandonne en grande partie la rhétorique orientalisante qui fleurissait sous sa plume sur la montagne, pour revenir plus simplement aux mots Dieu et Paradis, du lexique catholique... Il paraît se moquer lui-même de sa grande réponse d'adepte du Zen: Vous vous imaginez seulement que je suis fort ${ }^{14}$.»

\section{«He knew he wasn't... not to that extent...»}

«Carolyn, il rit de lui-même comme un vieux fou indulgent. L'idée du Vide semble régresser: le brouillard est le brouillard, les feuilles sont des feuilles, les sacs en papier sont des sacs en papier dont la pluie fera de la bouillie, les souches sont des souches, le sable est du sable: l'aspect matériel des choses est plus sensible. La vie est moléculaire, bien concrète. Il ne suffit plus à Kerouac de tout

11 Ibid., p. 41.

12 Joyce Johnson, Personnages secondaires, Paris, Sylvie Messinger, 1984,240 p.

13 Big Sur, op. cit., p. 34.

14 Ibid., p. 43. 
englober sous le vocable «Vide» pour se sentir apaisé. Et lorsqu'il voit ces feuilles d'arbres bien matérielles emportées par le ruissellement, il s'écrie: $O h$ mon Dieu, nous sommes tous balayés par la mer, quoi que nous sachions, quoi que nous disions ${ }^{15}$.»

«The ocean! My plane...»

«Encore une minute, Carolyn... Big Sur, comprends-tu, se trouve aux antipodes de Desolation Peak: sur les sommets rayonnait l'espérance; au pied des rochers, il n'y aucun espoir. C'est d'ailleurs la mer, Carolyn, qui cause la perte de ce vieil abstème malheureux de Kerouac.»

«Abstinence...?»

«Oui, Carolyn, écoute, c'est l'air de la mer, aspiré profondément, qui lui insuffle le malaise devant conduire à la crise de conscience qui provoquera le retour à la ville. La mer lui sussure: tu es un mort en puissance. Jack se détourne d'elle et de son influence mortifere. Si sur la montagne Jack était tombé en amour avec Dieu, la mer qui doit être comme Dieu ${ }^{16}$ le repousse et l'envoie seul consommer son destin. Rien ne sert de discourir. La mer est en lui, dans ses veines, et ce n'est que par nostalgie de cette béatitude originelle que connut la première cellule vivante que le vieux Canuck s'envoie du vin derrière la cravate. Jack est pendu à ses origines (Mémère, Lowell, Canada, Bretagne). Il a bénéficié de beaucoup de corde, mais la route n'était toujours que cette corde pour le pendre. D'accord?

\section{«Okay...Oh dear, my plane...»}

«Carolyn, Kerouac boit parce qu'il ne peut plus téter au sein flétri de Mémère. Sa sexualité n'a jamais pu s'affranchir du grand premier amour freudien sur lequel pèse l'interdit atavique. Encore une minute, Carolyn: la plénitude monacale de cette nouvelle tentative de vie solitaire est battue en brèche par Neptune lui-même: va te dissoudre dans le plaisir triste, mon bon Jack...»

\section{象}

«Apporte-moi une Black, mon bon Carl. Carolyn, nous touchons au but. Fini le lyrisme des bas-fonds. Dans Big Sur, il n'y a que la mort dans l'âme, la culpabilité immanente qui pèse de toute sa détermination sur le Destin. Ici encore, c'est une histoire de souris, tu suis, Carolyn? Comme Kerouac n'est pas chez lui, il ne peut prendre la liberté de retirer le raticide des tablettes où Monsanto l'a placé stratégiquement, en bon propriétaire soucieux de ses droits sur la nature. Kerouac se contente de laisser la boîte de poison hors de portée (du 
moins le croit-il). Mais lorsqu'il sera de retour avec les copains, déjà en train de sombrer dans la folie éthylique et l'obsession cruelle de la mort, il retrouve «sa» souris morte, victime indirecte de sa négligence coupable. Décidément, Kerouac n'échappe pas au meurtre de la souris. Pour Jack-in-the-Cabin, la souris qu'il nourrit tendrement apparaît comme un symbole de son âme fragile. À Desolation Peak, l'acte de tuer la souris était lié à la redescente sur terre, à la chute. À Big Sur, l'empoisonnement de la souris est encore une métaphore du mal. Le petit cadavre convulsé dans l'herbe, c'est une âme minée par l'alcool qui fait entrevoir sa mort au vieux Canuck.

«Enfin, Carolyn, les deux expériences érémitiques que sont Desolation Angels et Big Sur se situent sur le même axe, mais en sont les pôles opposés. Desolation Angels représente une aspiration céleste, une appréhension positive du Vide, une ouverture sur l'éternité dorée, une révélation de la liberté absolue. Big Sur, au contraire, est une plongée dans la pulsion de mort, une perception plus «matérialiste» de la mort vue comme anéantissement biologique, dissolution sans espoir dans l'océan originel. Si Kerouac a réussi à imprimer son être dans la géographie de son pays, on peut considérer que Desolation Peak représente son âme, alors que Big Sur tient lieu de corps périssable. Tandis qu'à Desolation Peak, le Vide Tout-Puissant appelle à la désincarnation de l'être, à Big Sur, l'océan organique réclame pesamment un corps que le ciel avait cru pouvoir lui soustraire.»

«I must come back to Québec! To hear the rest...»

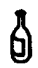

«Merci pour la Black, Maurice. Tu sais, je songe tout à coup, en regardant ces bouteilles de bière brunes, que le brun était la couleur de la vie chez Jack Kerouac. Et sur toutes nos bputeilles, l'étiquette noire est bien collée...»

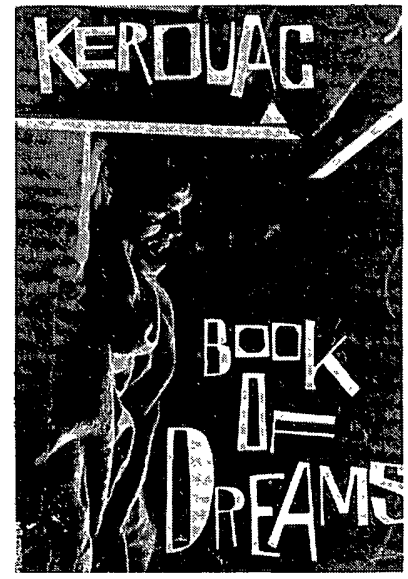

